\title{
Genome-wide association study leads to novel genetic insights into resistance to Aspergillus flavus in maize kernels
}

\author{
Guomin Han ${ }^{1,2}$, Cuiping Li1, Fangzhi Xiang1', Qianqian Zhao ${ }^{1}$, Yang Zhao ${ }^{1,2}$, Ronghao Cai', Beijiu Cheng ${ }^{1,2}$,
} Xuewen Wang $^{3^{*}}$ and Fang Tao ${ }^{1 *}$

\begin{abstract}
Background: Fungus infection in staple grains affects the food storage and threatens food security. The Aspergillus flavus is known to infect multiple grains and produce mycotoxin Aflatoxin B1, which is mutagenic, teratogenic and causes immunosuppression in animals. However, the molecular mechanism of maize resistance to $A$. flavus is largely unknown.

Results: Here we used corn kernels to investigate resistance genes to A. flavus using genome-wide association study (GWAS) of 313 inbred lines. We characterized the resistance levels of kernels after inoculating with $A$. flavus. The GWAS with 558,529 SNPs identified four associated loci involving 29 candidate genes that were linked to seed development, resistance or infection, and involved in signal pathways, seed development, germination, dormancy, epigenetic modification, and antimicrobial activity. In addition, a few candidate genes were also associated with several G-protein signaling and phytohormones that might involve in synergistic work conferring different resistance during seed development. Expression of 16 genes out of 29 during kernel development was also associated with resistance levels.

Conclusions: We characterized the resistance levels of 313 maize kernels after inoculating with A. flavus, and found four associated loci and 16 candidate maize genes. The expressed 16 genes involved in kernel structure and kernel composition most likely contribute to mature maize kernels' resistance to A. flavus, and in particular, in the development of pericarp. The linked candidate genes could be experimentally transformed to validate and manipulate fungal resistance. Thus this result adds value to maize kernels in breeding programs.
\end{abstract}

Keywords: Maize, Aspergillus pathogen, GWAS, SNP, Aflatoxin B1, Resistance

\section{Background}

Corn, also known as maize (Zea mays L.), is one of the most important cereal crops, grown throughout the world for both feed stock and food. Food security has been frequently threatened by toxic contamination produced by fungal infection [1]. Fungal species in

\footnotetext{
*Correspondence: xwwang@uga.edu; taofang@ahau.edu.cn

${ }^{3}$ Department of Genetics, University of Georgia, Athens 30602, USA

${ }^{1}$ School of Life Sciences, Anhui Agricultural University, Hefei 230036, China

Full list of author information is available at the end of the article
}

Aspergillus genus, e.g., soil borne A. flavus, are known to produce aflatoxins, a carcinogenic mycotoxin affecting human and animals [1]. Corn and other grains are susceptible to A. flavus infection at pre- and post-harvest, leading to accumulation of toxic aflatoxin $\mathrm{B} 1$ which is very stable and difficult to remove during processing [2]. To prevent corn kernels from A. flavus infection, continuous efforts have been made to reduce infection throughout the past 50 years. It is well known that some corn accessions, e.g., the Tuxpan in Mexico, possess

(c) The Author(s). 2020 Open Access This article is licensed under a Creative Commons Attribution 4.0 International License, which permits use, sharing, adaptation, distribution and reproduction in any medium or format, as long as you give appropriate credit to the original author(s) and the source, provide a link to the Creative Commons licence, and indicate if changes were made. The images or other third party material in this article are included in the article's Creative Commons licence, unless indicated otherwise in a credit line to the material. If material is not included in the article's Creative Commons licence and your intended use is not permitted by statutory regulation or exceeds the permitted use, you will need to obtain permission directly from the copyright holder. To view a copy of this licence, visit http://creativecommons.org/licenses/by/4.0/ The Creative Commons Public Domain Dedication waiver (http://creativecommons.org/publicdomain/zero/1.0/) applies to the data made available in this article, unless otherwise stated in a credit line to the data. 
high resistance to A. flavus and aflatoxin accumulation; therefore, incorporating high genetic resistance via breeding has been recognized as a promising solution. Several inbred lines with improved resistance to $A$. flavus through breeding selection have been reported by USDA-ARS and summarized in reviews [3-5]. Thus, it is of theoretical and applied importance to identify heritable genetic variants in maize conferring high resistance to A. flavus infection [3, 6].

Genes in plant-pathogen defense such as resistance (R) genes and signaling cascade genes should contribute to pathogen resistance in maize. However, resistance is usually regulated by multiple genes and interaction between maize and A. flavus, which results in difficulty in finding key genes. Using linkage mapping, quantitative trait loci (QTLs) were found on multiple chromosomes, e.g., 1, 2, 3, 4, 5, 9 in crossing populations derived from high resistance line Mp313E and susceptible line Va35 or B73 [7, 8]. Another study, using a genome-wide association study (GWAS) showed a significant locus at chromosome 8 with QTL in recombination inbred lines derived from parent line RA and M53 [6]. Two QTLs in bins 6.06 centimorgan $(\mathrm{cM})$ and $7.03 \mathrm{cM}$ were believed to be the most promising for the marker-assisted introgression of the resistance present in Mp715 [9]. However, those reported QTL for resistance to A. flavus are still in large chromosomal regions, usually several centimorgans. A major QTL qAA8 region on chromosome 8 for A. flavus resistance was confirmed and refined by using 228 recombinant inbred lines (RILs) and an association population comprising 437 maize inbred lines [6]. No significant QTL associated with aflatoxin accumulation was observed in an association population of 346 maize inbred lines which were testcrossed to stiff-stalk line Tx714 [10]. By comparison, GWAS analysis identified 107 SNPs associated with aflatoxin accumulation by using an association mapping panel of 300 inbred lines which were crossed to a susceptible line Va35 [11]. Some genes exhibit higher expression levels within QTL bins in the highly resistant line MP313E than susceptible lines revealed by microarray expression analysis [12]. Genes in the jasmonic acid biosynthesis pathway may be associated with resistance to A. flavus according to GWAS analysis [13]. A survey of candidate genes for maize resistance to infection by A. flavus and/or aflatoxin contamination demonstrated that none of the genes identified in past studies or in-house studies is significant [14].

To date, the reference sequence of maize genome is available in several varieties [15-19]. The genetic variance, especially the SNPs and InDels, has been made public in recent years with the advances of highthroughput sequencing technology, like DNA resequencing $[20,21]$. Li, et al. (2013) reported 556,944
SNPs in a panel of 368 maize accessions against maize B73 genome (version 2) [21]. Based on these DNA variants, several genotype-phenotype analyses have successfully discovered some gene loci via GWAS [22], e.g., ZmVPP1 encoding the pyrophosphatase which can improve drought tolerance [23], and ZmFBL41 conferring banded leaf phenotype and sheath blight resistance in maize revealed by using a natural variation population [24].

In this study, we investigated the difference in resistance to A. flavus infection in post-harvest corn kernels in a 313-diversity panel. Combining with SNP variance, we aimed to identify genomic region associated with the resistance to A. flavus via GWAS analysis. Further functional screening of genes flanking $80 \mathrm{~Kb}$ region allowed us to identify gene candidates responsible for the resistance difference. Expression of 16 genes out of the 29 candidates during seed development was also checked for association with resistance levels. We successfully uncovered the genetic variance at four loci including several novel loci in maize conferring the resistance to A. flavus at post-harvest, which could guide genetic breeding improvement in maize for storage.

\section{Results}

\section{Variations in kernel's resistance to pathogen $A$. flavus}

To examine the resistance to pathogen $A$. flavus infection, $\sim 24$ intact maize kernels from each of a panel consisting of 313 inbred lines $[20,21]$ were inoculated with $A$. flavus in a growth chamber for seven days. The rate of resistance was classified into eleven levels by measuring the ratio of infected area to the total kernel surface area (Fig. 1, Table S1). Thus, a lower infection ratio should reflect a higher resistance. We observed the variation in resistance to A. flavus, which follows a normal distribution. Sixty inbred lines with the highest frequency had an infection rate around 4. Seventeen and 20 lines had a higher infection ratio $(>9)$ and lower infection rate $(<1)$, respectively (Fig. 1). The susceptible kernels had visible mycelia inside while the kernels of resistant inbred lines did not (Fig. 2). Additionally, less Aflatoxin B1 was produced in the kernels of resistant inbred lines than that of susceptible lines (Fig. S1).

\section{Genetic loci associated with resistance to A. flavus via GWAS}

To investigate the associated gene candidates conferring the resistance to $A$. flavus at whole genome scale, we carried out a SNP-based GWAS. After filtration of minor allele frequency (MAF) $<0.05$, a total of 556,790 SNPs were used to identify the associated loci via the general linear model (GLM) and mixed linear model (MLM) [21, 25]. Association analysis via GLM identified four SNP regions significantly associated with resistance 


\section{(A)}

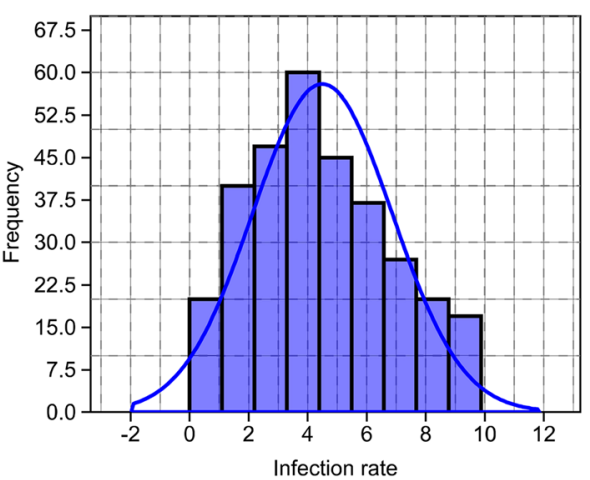

(B)

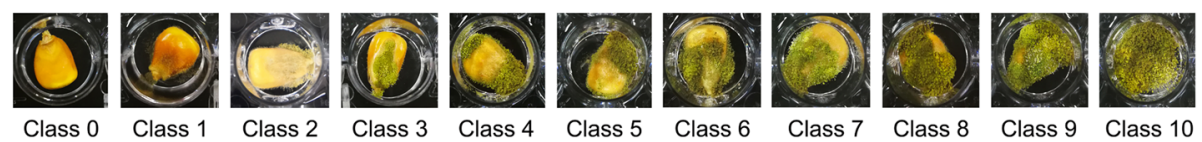

Fig. 1 Infection rate of A. flavus in maize kernels. (a) The image shows a histogram of inoculated infection rate of $A$. flavus on the surface of maize kernels of 313 inbred lines in a germplasm pool. The curve shows the normal distribution. (b) The images of infection rate at class 0 to 10

at the threshold of $p \leq 1.8 \times 10^{-6}$, distributed throughout four chromosomes (Fig. 3). The GWAS analysis detected that four, three, one, and three SNPs were associated with regions on chromosome 1, 2, 8 and 9, respectively (Table 1). Compared with GLM, only one SNP region was detected on chromosome 2 via MLM. Two associated SNPs on chromosome 2 were the same in regard to the SNPs that were identified by GLM (Table 1). Fewer associated SNPs were detected via MLM than that of GLM (Fig. 3). Here, the results of GLM were used for subsequent analyses.

\section{Candidate genes in maize confer resistance to pathogen} A. flavus

The SNP loci identified by GWAS provide clues for understanding the genetic architecture which confers the variation in resistance. The maize inbred lines and SNP data have been used to investigate oil biosynthesis, drought tolerance, banded leaf and sheath blight resistance, etc., and $50 \mathrm{~kb}$ to $100 \mathrm{~kb}$ region of the peak SNP were often used to search candidate genes [21-24]. To resolve candidate genes responsible for $A$. flavus resistance, we further extracted the genes flanking $80 \mathrm{~kb}$ at either side of the associated SNP locus and within the significant associated region, and then annotated their functions against databases to identify the most likely gene candidates. After comparing the annotation of genes in maize genome assembly between version V2 and V4 (Table S2), we kept the consistent genes and the genes with additional evidence as the final gene set. Nine, nine, nine and two candidate genes were associated with two, two, one, and one SNP region on chromosome 1, 2, 8 and 9, respectively (Table 1). Further boxplot analyses showed that the polymorphisms of

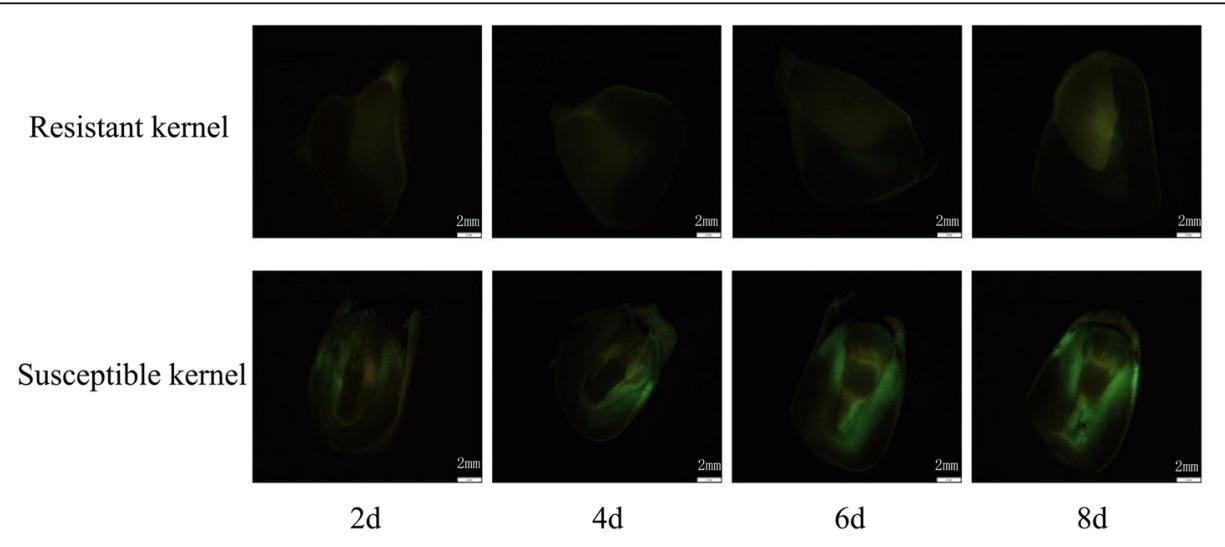

Fig. 2 Comparison the penetration processes of $A$. flavus hyphae into different maize kernels. $2 d, 2$ days after inoculation with $A$. flavus conidia; $4 d, 4$ days after inoculation with $A$. flavus conidia; $6 \mathrm{~d}, 6$ days after inoculation with $A$. flavus conidia; $8 d$, 8 days after inoculation with $A$. flavus conidia 

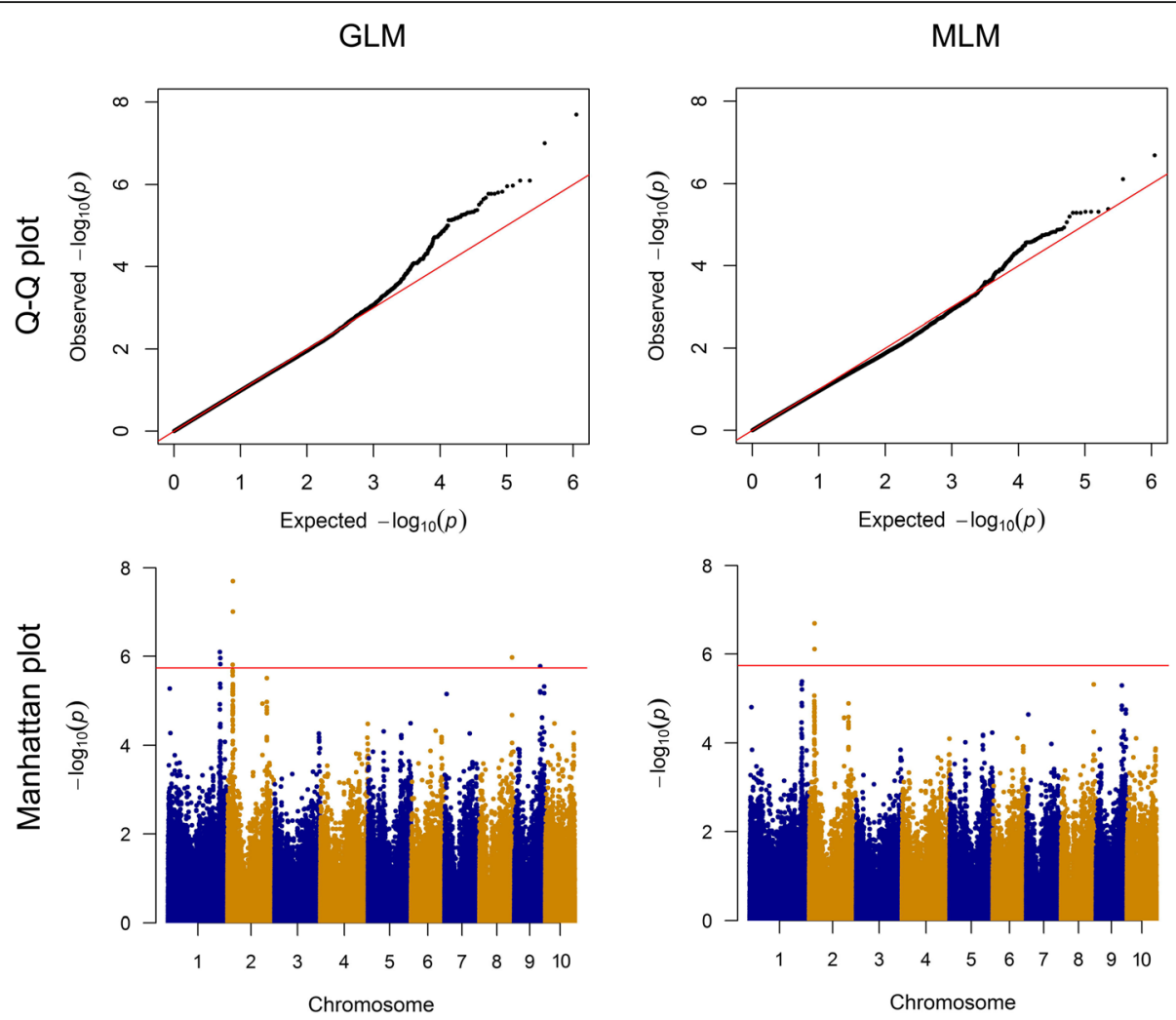

Fig. 3 Q-Q plot and Manhattan plot showing associated loci for resistance to A. flavus on maize kernels via the general linear model (GLM) and mixed linear model (MLM). The SNP locus was plotted in dots and the loci above the red line are considered to be significant at threshold of $-\log 10(p)>1.8 \times 10^{-6}$

all six SNPs fit well with the variation of each resistance ranks (Fig. 4), which suggested that the six SNPs are likely associated with genes conferring the resistance to A. flavus.

\section{Relation of gene expression and resistance to A. flavus in the germplasm panel}

To indentify the expression of each candidate gene, raw transcriptome profiles in the developmental maize kernel of 368 inbred lines at 15 days after pollination (DAP) were downloaded from NCBI and analyzed with the HISAT2 tool. Results show that the majority of resistance associated genes were expressed during the development of kernel in inbred lines with the exception of genes GRMZM2G024131, GRMZM2G319150, and GRMZM2G474575 which were not, or were very lowly expressed, in all inbred lines (Fig. 5 and Table S3). The abundance of transcripts expressed from these genes was used to conduct a SNP-expression association analysis via GWAS [20]. Consistent with resistance-SNP association results, sixteen out of 29 genes were associated with the flanking $80 \mathrm{~kb}$ region (Table 1), indicating that the sixteen expressed genes involving in kernel structure and kernel composition are most likely contributing to mature maize kernels' resistance to A. flavus.

It is worth noting that three adjacent SNPs (chr9.S 131,725,983, chr9.S_131,726,004, and chr9.S_131,726, 006) on chromosome 9 in this study overlapped with a loci previously identified in pre-harvest resistance to $A$. flavus in a study conducted by Kelley, et al (2012) (Fig. 6) [12], indicating a common genomic feature for resistance. In this region, gene GRMZM2G309327 and GRMZM2G108655 are the most important candidate genes in pre-harvest investigations. The gene GRMZM2G309327, as a homolog to AT4G20780 and AT5G44460 in Arabidopsis, encodes Calcium-binding protein (CML42) which is known to function in signal transportation during infective defense in plant $[26,27]$. This gene is also expressed in the pericarp/aleurone of the corn kernel [28]. The gene GRMZM2G108655 encodes COP1-interacting protein 7 involving in anthocyanin biosynthesis [29], indicating that the gene can influence seed coloration.

Besides the three adjacent SNPs on chromosome 9, the remaining associated SNPs are newly identified in the association results to A. flavus resistance. Many candidate genes were reported to be involved in seed 
Table 1 Genetic loci and candidate genes associated with resistance to A. flavus identified via GWAS

\begin{tabular}{|c|c|c|c|c|c|c|c|c|}
\hline Marker & Chrom & Locus_pos & $P$ value & $\mathrm{R} 2(\%)$ & $\begin{array}{l}\text { Candidate } \\
\text { Gene Id }\end{array}$ & Annotated in NR of NCBI & Expressed or not & $\begin{array}{l}\text { Verified by } \\
\text { expression } \\
\text { GWAS }\end{array}$ \\
\hline \multirow[t]{5}{*}{ chr1.S_262891312 } & 1 & 262891312 & $8.05 \mathrm{E}-07$ & 11 & GRMZM2G113569 & histone H3.v1 & expressed & TRUE \\
\hline & & & & & GRMZM2G085116 & Protein of unknown function, DUF538 & expressed & TRUE \\
\hline & & & & & GRMZM2G024131 & indole-3-acetate beta-glucosyltransferase & Not expressed & \\
\hline & & & & & GRMZM2G319150 & Ferredoxin--NADP reductase, chloroplastic & Not expressed & \\
\hline & & & & & GRMZM2G122018 & germin-like protein 2 precursor & expressed & \\
\hline chr1.S_262891333 & 1 & 262891333 & $8.05 \mathrm{E}-07$ & 11 & - & & & \\
\hline \multirow[t]{4}{*}{ PZE-101214474 } & 1 & 264954323 & $1.50 \mathrm{E}-06$ & 11 & GRMZM2G346263 & opaque endosperm10 & expressed & \\
\hline & & & & & GRMZM2G044557 & $\begin{array}{l}\text { mitogen-activated protein kinase kinasekinase } \\
1 \text {-like isoform } x 1\end{array}$ & expressed & \\
\hline & & & & & GRMZM2G400092 & histone acetyltransferase hat $b$ & expressed & TRUE \\
\hline & & & & & GRMZM2G042133 & DnaJ & expressed & TRUE \\
\hline chr1.S_264955840 & 1 & 264955840 & $1.10 \mathrm{E}-06$ & 11 & - & & & \\
\hline \multirow[t]{3}{*}{ chr2.S_26290253 } & 2 & 26290253 & $1.56 \mathrm{E}-06$ & 11 & GRMZM2G127581 & tubulin-specific chaperone B & expressed & TRUE \\
\hline & & & & & GRMZM2G127591 & udp-galactose transporter 1-like & expressed & \\
\hline & & & & & GRMZM2G428386 & $\begin{array}{l}\text { 1-phosphatidylinositol-3-phosphate 5-kinase } \\
\text { FAB1D }\end{array}$ & expressed & TRUE \\
\hline \multirow[t]{6}{*}{ chr2.S_26717856 } & 2 & 26717856 & $9.83 \mathrm{E}-08$ & 13 & GRMZM2G113990 & $\begin{array}{l}\text { coiled-coil-helix-coiled-coil-helix } \\
\text { domain-containing protein } 4\end{array}$ & expressed & \\
\hline & & & & & GRMZM2G114149 & Mitochondrial substrate carrier family protein & expressed & TRUE \\
\hline & & & & & GRMZM2G114153 & gem-like protein & expressed & TRUE \\
\hline & & & & & GRMZM2G413887 & gem-like protein & expressed & TRUE \\
\hline & & & & & GRMZM2G474575 & alpha-amylase/subtilisin inhibitor & Not expressed & \\
\hline & & & & & GRMZM2G171410 & mrna cap guanine-n 7 methyltransferase 2 -like & expressed & TRUE \\
\hline chr2.S_26718237 & 2 & 26718237 & $2.02 \mathrm{E}-08$ & 14 & - & & & \\
\hline \multirow[t]{9}{*}{ chr8.S_165276011 } & 8 & 165276011 & $1.06 \mathrm{E}-06$ & 11 & GRMZM2G431066 & auxin-responsive protein SAUR50 & expressed & \\
\hline & & & & & GRMZM2G129065 & protein trichome berefringence-like 7 & expressed & \\
\hline & & & & & GRMZM2G002023 & $\begin{array}{l}\text { udp-glucuronate:xylan } \\
\text { alpha-glucuronosyltransferase 1-like }\end{array}$ & expressed & \\
\hline & & & & & GRMZM2G092759 & N-lysine methyltransferase setd 6 & expressed & TRUE \\
\hline & & & & & GRMZM2G393070 & dirigent protein 8 -like & expressed & TRUE \\
\hline & & & & & GRMZM2G393072 & dirigent protein 9-like & expressed & TRUE \\
\hline & & & & & GRMZM2G092817 & 4,5-DOPA dioxygenase extradiol & expressed & TRUE \\
\hline & & & & & GRMZM2G092945 & amino acid permease 2 & expressed & TRUE \\
\hline & & & & & GRMZM2G096407 & amino acid permease 8 -like & expressed & \\
\hline \multirow[t]{2}{*}{ chr9.S_131725983 } & 9 & 131725983 & $1.67 \mathrm{E}-06$ & 11 & GRMZM2G309327 & Calcium-binding protein CML42 & expressed & \\
\hline & & & & & GRMZM2G108655 & COP1-interacting protein 7 & expressed & TRUE \\
\hline chr9.S_131726004 & 9 & 131726004 & $1.67 \mathrm{E}-06$ & 11 & - & & & \\
\hline chr9.S 131726006 & 9 & 131726006 & $1.67 \mathrm{E}-06$ & 11 & - & & & \\
\hline
\end{tabular}

Note: The black SNP markers, SNPs detected only using GLM; the red markers, SNPs detected using GLM and MLM

development. The associated loci on Chr2 is close to previously reported QTL loci resistant to A. flavus [7]. At the loci (Table 1), gene GRMZM2G127591 is homologous to Arabidopsis AT1G21870, encoding GOLGI NUCLEOTIDE SUGAR TRANSPORTER 5, which has been reported to be required for synthesis of essential cell surface component [30]. Among the genes at loci on Chr8, gene GRMZM2G129065 encodes a TRICHOME BIREFRINGENCE-LIKE protein, which is homologous to Arabidopsis gene AT1G48880. This gene is involved in the synthesis and deposition of secondary wall cellulose by influencing the esterification state of pectic polymers [31, 32]. The gene GRMZM2G400092 encodes a histone acetyltransferase (HAT) B which is involved in acetylation of newly synthesized histone $\mathrm{H} 4$ during embryo germination. HAT B is also involved in seed development and germination in maize [33, 34]. GRMZM2G393070 and GRMZM2G393072 are dirigent proteins which were reported to mediate lignan and cyanogenic glucoside formation in Flax seed [35]. 


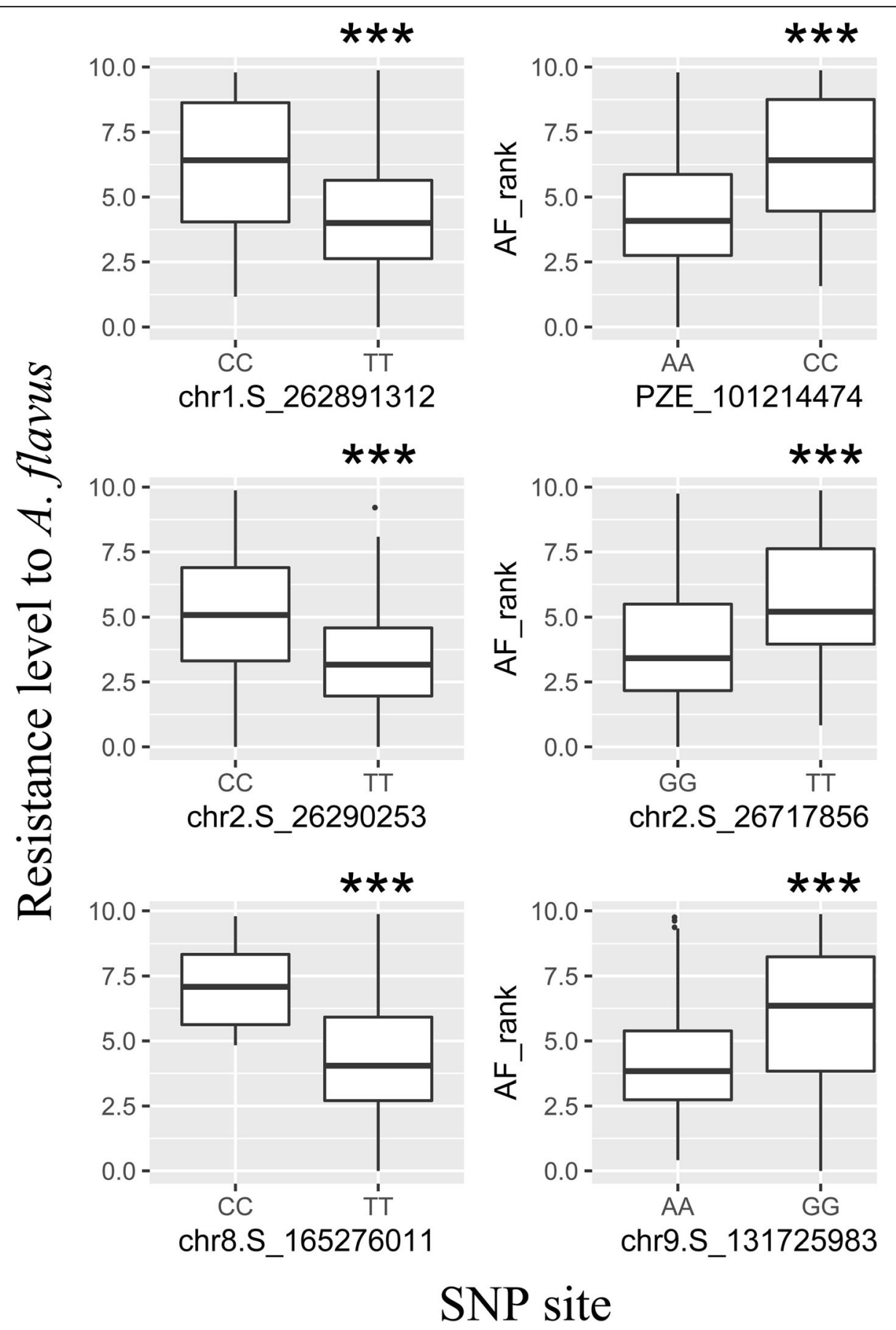

Fig. 4 Boxplot of resistance rank to A. flavus at significantly associated SNPs. The resistance levels of all inbred lines at vertical axis were boxplotted along the SNP alleles at the horizontal axis, ${ }^{* * *}$ indicates $p<0.001$ via T-test

\section{Discussion}

The seed coat of flowering plants plays important roles in seed germination, vigor and longevity potentials [36]. It is also the first surface of seeds' defense against adverse external factors [37]. The seed coat of maize kernel contains two outer layers of the kernel, called the pericarp and the aleurone. Approximately $87 \%$ of the pericarp is crude fiber which is mainly constituted of hemicellulose (67\%), cellulose (23\%) and lignin (0.1\%) [38]. The tough fiber of pericarp forms a shield to protect the interior of seed from abiotic and biotic stresses including infection of mold and bacteria [36, 39]. Once A. flavus penetrates the seed coat or pericarp of kernels, the fungal mycelia can grow inside the kernel of 


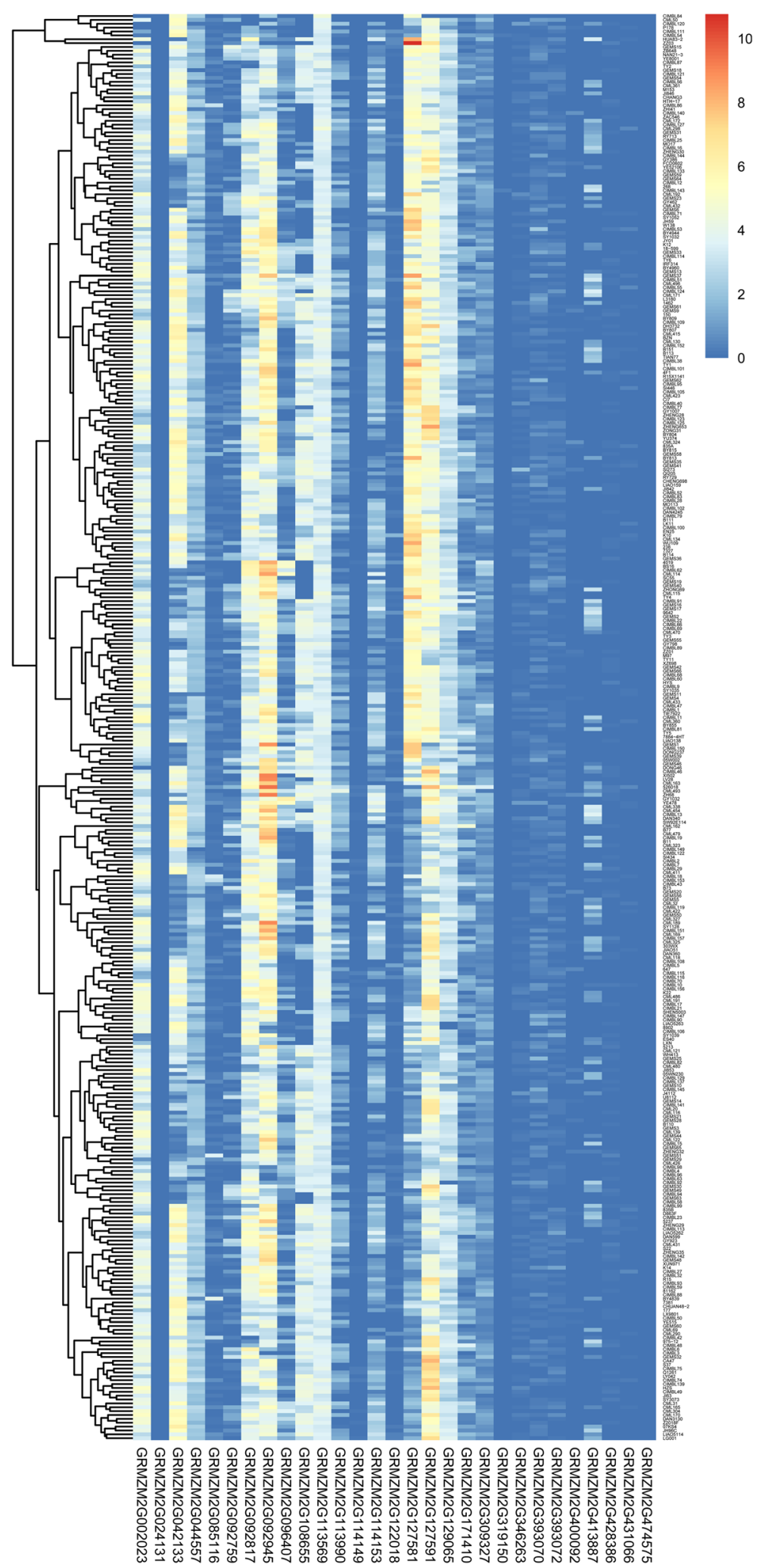

Fig. 5 The expression levels of the candidate genes in 368 inbred lines. The expression data are from seed tissue at 15 days old after pollination in the study of Fu et al. (2013) 


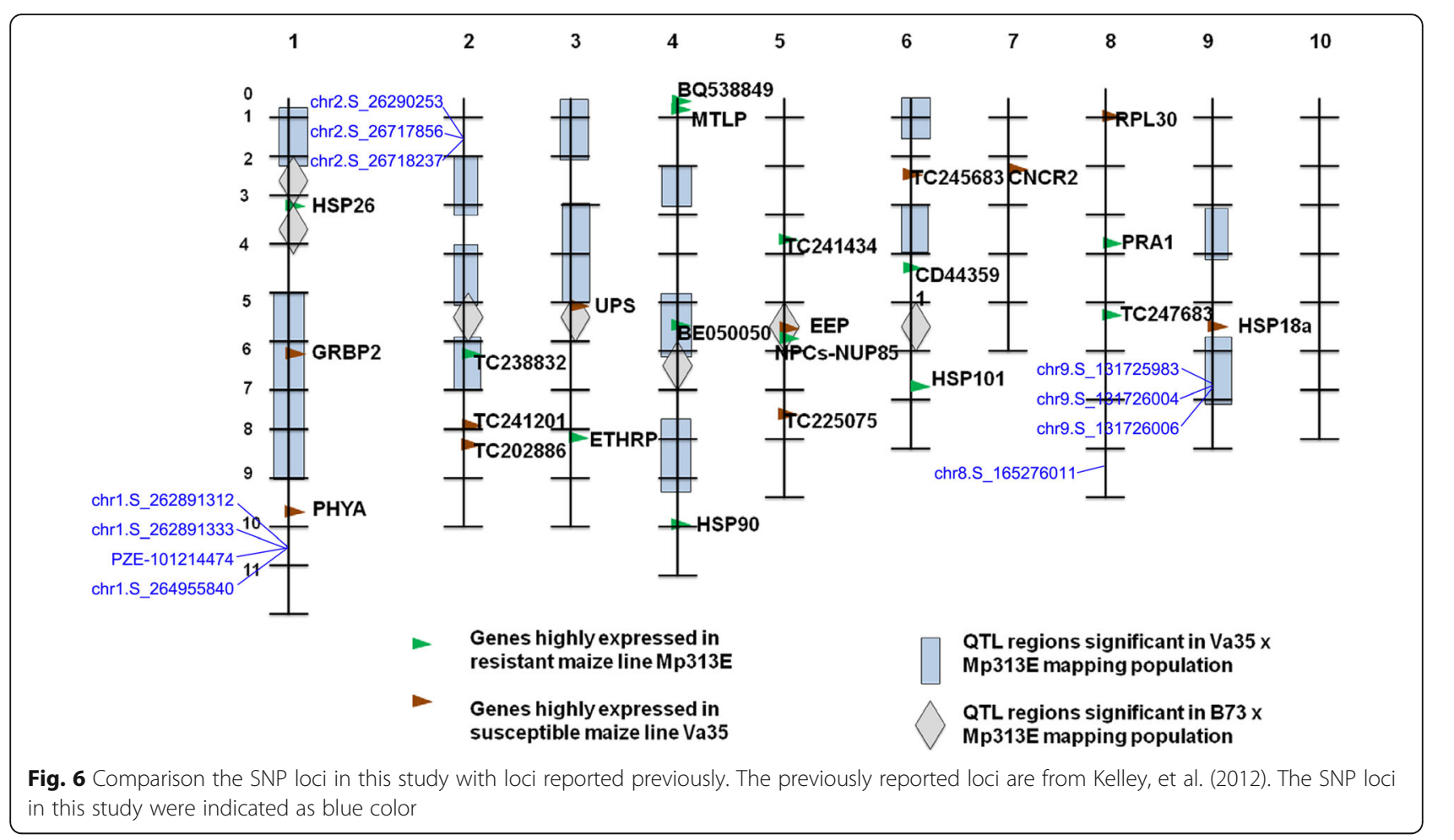

susceptible inbred lines (Fig. 2). Generally, the more the fungi penetrated into the maize kernels, the higher Aflatoxin B1 was detected in kernels post 7 days of inoculation with A. flavus conidia (Fig. S1). The results indicated that the intactness of maize seeds should play a vital role in resistance to $A$. flavus infection in postharvest corn kernels.

The seed coat also acts as channel for transmitting environmental cues, such as water into the interior of the seed in addition to as a shield [37]. After absorbing water, maize seeds will swell. The integrity of the pericarp should play a vital role in the kernel resistance to mold, and the rate of kernel resistance to A. flavus reflects the integrity of seed after assimilation of water. The diversity of the resistance levels in this study indicated that the integrity of seeds of inbred lines should be different after absorbing water. Among the inbred lines, results of 17 seed lines with a higher infection ratio $(>9)$ while 20 seed lines with a lower infection rate $(<1)$ suggest that the lines with lower infection rate $(<1)$ were more intact than that of lines with a higher infection ratio (>9) during the fungal infection process. In this study, two, two, one, and one SNPs with 4, 6, 5 and 1 candidate genes, which were further verified by SNPexpression GWAS analysis, were associated with regions on chromosome 1, 2, 8 and 9, respectively. The six SNPs could explain $11 \sim 14 \%$ of the phenotypic variation. The majority of genetic loci associated with resistance to $A$. flavus in this study were different from the QTL regions in the study of Kelley, et al (2012) [12], which might be due to the fact that different development stages and storage condition of seeds were used. Fourteen-day-old seeds after self-pollination were inoculated with the fungus A. flavus strain NRRL 3357 in their study [12] while dry mature seeds were used in our study, which should contribute to the difference.

The intactness of maize seeds after absorbing little water might be controlled by the integrity of seed coat and the moisture absorption capacity of the endosperm. Seeds with cracking or broken seed coats are prone to mold infection [37, 40, 41]. A mature seed is developed from the zygote which is double fertilized, resulting in the formation of a diploid embryo and a triploid endosperm [37, 42]. In contrast to the embryo and endosperm, the seed coat is entirely maternal in origin [37]. To understand kernel development, immature seeds of 368 inbred lines at 15 DAP were investigated by RNA sequencing [20]. The gene expression profile of the lines is highly variable, and relationships between expression quantitative trait loci (eQTL) and their targets were established to reveal gene regulatory networks [20]. Although the expression levels of the candidate genes in maize kernels without $A$. flavus inoculation should not be directly involved in fungal resistance during kernel development, at least some of the expressed genes could be the candidate genes contributing to the different components of kernels. It has been summarized that seed size is determined by several pathways including the IKU pathway, the ubiquitin-proteasome pathway, G-protein signaling, the mitogen-activated protein 
kinase signaling pathway, phytohormones and transcriptional regulatory factors [42]. The pathways can influence the formation of seed size by regulating the endosperm and/or maternal tissue growth. In this study, some candidate genes belong to G-protein signaling pathways (GRMZM2G113569, and GRMZM2G309327), while other genes are related to phytohormones (GRMZM2G431066, GRMZM2G114153, GRMZM2G413887 and GRMZM2G092759). The genes involved in the development of seed size were also the associated genes which contributed to A. flavus resistance. In addition, several associated genes were reported to be involved in the synthesis of the embryo, the endosperm, and seed coat. For example, GRMZM2G127591 and GRMZM2G092945 are engaged in the transportation of sugars and amino acids during seed development [30, 43]. GRMZM2G129065, GRMZM2G393070 and GRMZM2G393072 might be involved in the syntheses of polymers, e.g., pectin, lignin $[31,32,35]$. The G-protein signaling and phytohormones related genes and other candidate genes should synergistically work for the seed development, resulting in mature seeds with different capacity against adverse environmental conditions. Some seeds of the inbred lines will be of high resistance to mold infection, including A. flavus. The validation and further characterization of the detailed roles of likely gene candidates during the response to A. flavus infection could be of high priority in future experiments, such as gene overexpression and gene knockout analysis.

\section{Conclusions}

We characterized the resistance levels of 313 maize kernels after inoculating with $A$. flavus. Four loci and 16 candidate maize genes were mined via GWAS. The 16 expressed genes involving in kernel structure and kernel composition most likely contributed to resistance of mature maize kernels to A. flavus, particularly, genes involving in the regulating development of pericarp. The linked candidate genes could be experimentally transformed to validate and manipulate resistance to add value to maize kernels in breeding programs.

\section{Methods}

\section{Plant materials}

Three hundred and thirteen maize inbred lines, including tropical/subtropical inbred lines, temperate lines and lines of mixed origin, were kindly provided by Prof. Guoying Wang (Institute of Crop Science, Chinese Academy of Agricultural Sciences). All the inbred lines were planted in the field of Sanya (Hainan Province, $18.75 \mathrm{~N}, 109.17 \mathrm{E})$ in November 2015. The mature seeds of each inbred line were used for fungal infection analysis.

\section{Analysis of maize resistance to fungal infection}

A laboratory kernel-screening assay (KSA) was performed as described by Brown et al. (1999) with modifications [44]. Briefly, the surface of $\sim 24$ kernels from each inbred line was sterilized with $75 \%$ ethanol and $1 \%$ $\mathrm{NaClO}$ for 5 min and dipped into a suspension of $A$. flavus conidia $\left(4 \times 10^{6} \mathrm{cfu} / \mathrm{mL}\right)$ for $5 \mathrm{~min}$. Kernels were then placed in a 24-well plate individually, one kernel in each well, and incubated at $28^{\circ} \mathrm{C}$ for 7 days. High humidity (>95\% RH) was maintained by supplying sterile water to the space between holes. Two biological replicate experiments were conducted for each inbred line. Infection was designed as visible mycelial and conidia on the surface of the kernel, and the infection was classified by the ratio of the infected area to the surface area of each kernel. Eleven classes were defined as follows: $0=$ no visible mycelial and conidia, $1=1-10 \%, 2=21-30 \%$, $3=31-40 \%, 4=41-50 \%, 5=51-60 \%, 6=61-70 \%, 7=$ $70-80 \%, 8=80-90 \%, 9=90-100 \%, 10=100 \%$.

Aflatoxin B1 was extracted and the abundance of Aflatoxin B1 was determined by HPLC with a fluorescence detector [2]. The kernels were dried for two days in a $60^{\circ} \mathrm{C}$ oven and ground into powder. The Aflatoxin B1 were extracted in methanol: $\mathrm{H}_{2} \mathrm{O}=70: 30(\mathrm{v} / \mathrm{v})$, shaking at $190 \mathrm{rpm}$ at $20^{\circ} \mathrm{C}$ for two hours, followed by centrifuging for five mins at $8000 \mathrm{rpm}$. The supernatant was filtered through $0.22 \mu \mathrm{m}$ filter and filter-through liquid was used for HPLC analysis.

\section{Genome-wide association study and annotation of candidate genes}

558,529 SNPs, population structure and kinship data were kindly provided by Prof. Guoying Wang and Prof. Jianbing Yan (http://www.maizego.org) [20, 21]. 558,529 SNPs in the maize germplasm pool were used to carry out GWAS with TASSEL (v5.0) [21, 25]. General Linear Model (GLM) and Mixed Linear Model (MLM) were used to identify the genetic loci associated with resistance to A. flavus infection as described previously [23]. To balance false negative and false positive associations in GWAS, two workflows were used: GLM plus principal component analysis (PCA) for population structure inference and principle components 1 and 2 as covariates, and MLM plus PCA as well as kinship by using Tassel 5 workflows. In addition, we also used the previous generated population structure information and kinship data to conduct GWAS $[20,21]$. Both input data sets yielded the same results. Manhattan and Q-Q plots were generated via the 'qqman' package in $R$. If the $P$-value was lower than Bonferroni-adjusted significance threshold $\left(1.8 \times 10^{-6}\right)$, the SNP was considered significant locus 
[21]. All the genes within $80 \mathrm{~kb}$ flanking region either upstream or downstream of the significant SNP site were further annotated via blastp against $\mathrm{Nr}$ of NCBI, InterProScan (swiss-prot), and Pfam [45, 46].

\section{Analysis of the expression level of candidate genes} The raw transcriptome profiles (BioProject: PRJNA208608) of maize kernels at 15 days after pollination were obtained from NCBI. The SRA format data were transformed into fastq format via NCBI fastq-dump in sratoolkit (version 2.9.2). The raw reads were trimmed to remove low quality base-calls and adaptor sequences using the Trimmomatic (v0.33) tool [47]. Cleaned reads were mapped to the genome of Z. mays B73 (V2) via HISAT2 (v2.1.0) [48]. StringTie was applied to assemble and integrate the transcripts in each sample [48]. The expression level of each gene was calculated by Ballgown [48]. The expression of each candidate gene was used to carry out GWAS again to search for SNP-expression association via TASSEL (v5.0) [25].

\section{Supplementary information}

Supplementary information accompanies this paper at https://doi.org/10. 1186/s12870-020-02404-5.

Additional file 1 Fig. S1 The content of Aflatoxin B1 in kernel of some maize lines after 7 days of inoculation with A. flavus. Table S1 Resistance level of the inbred lines. Table S2 Comparison of the protein IDs in V2 version with that in V4 version of maize B73. Table S3 The expression levels of the candidate genes in 368 inbred lines

\section{Abbreviations}

GWAS: Genome-wide association study; QTL: Quantitative trait loci; MAF: Minor allele frequency; ABA: Abscisic acid; HAT: Histone acetyltransferase; KSA: Kernel-screening assay; GLM: General Linear Model; MLM: Mixed Linear Model

\section{Acknowledgments}

The maize inbred lines were kindly provided by Prof. Guoying Wang. Thanks to Prof. Jianbing Yan and members from his laboratory for technical help. We are grateful to two anonymous referees for their helpful comments to improve this manuscript.

\section{Authors' contributions}

F. T., B. C. and X.W. conceived the study. F. T. and X.W. designed the study. F. T., C. L., F. X. and Q. Z. performed the experiments. Y. Z., R. C. and B. C. collected and propagated plant material. Y. Z., R. C. and B. C. revised the article. G. H. and X.W. analyzed and interpreted the data. X.W. and G.H. wrote the manuscript. All authors reviewed and approved the final manuscript.

\section{Funding}

This work was supported by the National Key Research and Development Program of China (Project No. 2017YFD0301306), the Educational Commission of Anhui Province of China (Project No. KJ2016A841). The authors declare that none of the funding bodies have any role in the research design, the data collection and analysis, and the manuscript preparation.

\section{Availability of data and materials}

The SNP data is available at http://www.maizego.org/Resources.html. The file name of the SNP data is 'SNPs_368lines_mafo.05_0.56 M.hmp.gz' at https:// pan.baidu.com/s/1t_S_kof4S8G1bj_j4_c4bQ\#list/path=\%2FMaizeGo\%2 OResources\%2FGenotype [20, 21]. All other data and material used in this study are available from the corresponding author upon reasonable request.
Ethics approval and consent to participate

Not applicable.

\section{Consent for publication}

Not applicable.

\section{Competing interests}

The authors declare that they have no competing interests.

\section{Author details}

${ }^{1}$ School of Life Sciences, Anhui Agricultural University, Hefei 230036, China. ${ }^{2}$ National Engineering Laboratory of Crop Stress Resistance Breeding, Anhui Agricultural University, Hefei 230036, China. ${ }^{3}$ Department of Genetics, University of Georgia, Athens 30602, USA.

Received: 15 October 2019 Accepted: 26 April 2020

Published online: 11 May 2020

\section{References}

1. Kumar P, Mahato DK, Kamle M, Mohanta TK, Kang SG. Aflatoxins: a global concern for food safety, human health and their management. Front Microbiol. 2016;7:2170

2. Han G, Shao Q, Li C, Zhao K, Jiang L, Fan J, Jiang H, Tao F. An efficient agrobacterium-mediated transformation method for aflatoxin generation fungus Aspergillus flavus. J Microbiol. 2018;56:356-64.

3. Shan X, Williams WP. Toward elucidation of genetic and functional genetic mechanisms in corn host resistance to Aspergillus flavus infection and aflatoxin contamination. Front Microbiol. 2014;5:364.

4. Spencer Smith J, Paul Williams W, Windham GL. Aflatoxin in maize: a review of the early literature from "moldy-corn toxicosis" to the genetics of aflatoxin accumulation resistance. Mycotoxin Res. 2019;35:111-28.

5. Fountain JC, Abbas HK, Scully BT, Li H, Lee RD, Kemerait RC, Guo B. Evaluation of maize inbred lines and topcross progeny for resistance to preharvest aflatoxin contamination. The Crop Journal. 2019;7:118-25.

6. Zhang Y, Cui M, Zhang JM, Zhang L, Li CL, Kan X, Sun Q, Deng DX, Yin ZT. Confirmation and Fine Mapping of a Major QTL for Aflatoxin Resistance in Maize Using a Combination of Linkage and Association Mapping. Toxins (Basel). 2016; 8.

7. Brooks TD, Williams WP, Windham GL, Willcox MC, Abbas HK. Quantitative trait loci contributing resistance to aflatoxin accumulation in the maize inbred Mp313E. Crop Sci. 2005;45:171-4

8. Willcox MC, Davis GL, Warburton ML, Windham GL, Abbas HK, Betran J, Holland JB, Williams WP. Confirming quantitative trait loci for aflatoxin resistance from Mp313E in different genetic backgrounds. Mol Breed. 2013; 32:15-26.

9. Smith JS, Williams WP, Windham GL, Xu W, Warburton ML, Bhattramakki D. Identification of quantitative trait loci contributing resistance to aflatoxin accumulation in maize inbred Mp715. Mol Breed. 2019;39.

10. Farfan ID, De La Fuente GN, Murray SC, Isakeit T, Huang PC, Warburton M, Williams P, Windham GL, Kolomiets M. Genome wide association study for drought, aflatoxin resistance, and important agronomic traits of maize hybrids in the sub-tropics. PLoS One. 2015;10:e0117737.

11. Warburton ML, Tang JD, Windham GL, Hawkins LK, Murray SC, Xu WW, Boykin D, Perkins A, Williams WP. Genome-wide association mapping of Aspergillus flavus and Aflatoxin accumulation resistance in maize. Crop Sci. 2015;55:1857-67.

12. Kelley RY, Williams WP, Mylroie JE, Boykin DL, Harper JW, Windham GL, Ankala A, Shan XY. Identification of maize genes associated with host plant resistance or susceptibility to Aspergillus flavus infection and Aflatoxin accumulation. PLoS One. 2012;7.

13. Tang JD, Perkins A, Williams WP, Warburton ML. Using genome-wide associations to identify metabolic pathways involved in maize aflatoxin accumulation resistance. BMC Genomics. 2015;16.

14. Hawkins L, Warburton M, Tang J, Tomashek J, Alves Oliveira D, Ogunola O, Smith J, Williams W. Survey of Candidate Genes for Maize Resistance to Infection by Aspergillus flavus and/or Aflatoxin Contamination. Toxins (Basel). 2018; 10:61.

15. Li C, Song W, Luo Y, Gao S, Zhang R, Shi Z, Wang X, Wang R, Wang F, Wang $J$ et al. The HuangZaoSi Maize Genome Provides Insights into Genomic Variation and Improvement History of Maize. \#N/A. 2019; 12:402-409. 
16. Schnable PS, Ware D, Fulton RS, Stein JC, Wei FS, Pasternak S, Liang CZ, Zhang JW, Fulton L, Graves TA, et al. The B73 maize genome: complexity, diversity, and dynamics. Science. 2009;326:1112-5.

17. Springer NM, Anderson SN, Andorf CM, Ahern KR, Bai F, Barad O, Barbazuk WB, Bass HW, Baruch K, Ben-Zvi G et al. The maize W22 genome provides a foundation for functional genomics and transposon biology. Nat Genet. 2018; 50:1282-+

18. Sun SL, Zhou YS, Chen J, Shi JP, Zhao HM, Zhao HN, Song WB, Zhang M, Cui $Y$, Dong XM et al. Extensive intraspecific gene order and gene structural variations between Mo17 and other maize genomes. Nat Genet. 2018; 50: 1289-+.

19. Yang N, Liu J, Gao Q, Gui ST, Chen L, Yang LF, Huang J, Deng TQ, Luo JY, He $L J$ et al. Genome assembly of a tropical maize inbred line provides insights into structural variation and crop improvement. Nat Genet. 2019; 51:1052-+.

20. Fu J, Cheng Y, Linghu J, Yang X, Kang L, Zhang Z, Zhang J, He C, Du X, Peng $Z$, et al. RNA sequencing reveals the complex regulatory network in the maize kernel. Nat Commun. 2013;4:2832.

21. Li H, Peng Z, Yang X, Wang W, Fu J, Wang J, Han Y, Chai Y, Guo T, Yang N, et al. Genome-wide association study dissects the genetic architecture of oil biosynthesis in maize kernels. Nat Genet. 2013;45:43-50.

22. Xiao Y, Liu H, Wu L, Warburton M, Yan J. Genome-wide association studies in maize: praise and stargaze. Mol Plant. 2017;10:359-74.

23. Wang X, Wang H, Liu S, Ferjani A, Li J, Yan J, Yang X, Qin F. Genetic variation in ZmVPP1 contributes to drought tolerance in maize seedlings. Nat Genet. 2016:48:1233-41.

24. Li N, Lin B, Wang H, Li X, Yang F, Ding X, Yan J, Chu Z. Natural variation in ZmFBL41 confers banded leaf and sheath blight resistance in maize. Nat Genet. 2019;51:1540-8.

25. Bradbury PJ, Zhang Z, Kroon DE, Casstevens TM, Ramdoss Y, Buckler ES. TASSEL: software for association mapping of complex traits in diverse samples. Bioinformatics. 2007;23:2633-5.

26. Ascencio-lbanez JT, Sozzani R, Lee TJ, Chu TM, Wolfinger RD, Cella R, Hanley-Bowdoin L. Global analysis of Arabidopsis gene expression uncovers a complex array of changes impacting pathogen response and cell cycle during geminivirus infection. Plant Physiol. 2008;148:436-54.

27. Chiasson D, Ekengren SK, Martin GB, Dobney SL, Snedden WA. Calmodulinlike proteins from Arabidopsis and tomato are involved in host defense against Pseudomonas syringae pv. Tomato. Plant Mol Biol. 2005;58:887-97.

28. Walley JW, Sartor RC, Shen Z, Schmitz RJ, Wu KJ, Urich MA, Nery JR, Smith LG, Schnable JC, Ecker JR, et al. Integration of omic networks in a developmental atlas of maize. Science. 2016:353:814-8.

29. Yamamoto $Y Y$, Matsui $M$, Ang LH, Deng XW. Role of a COP1 interactive protein in mediating light-regulated gene expression in arabidopsis. Plant Cell. 1998:10:1083-94.

30. Handford MG, Sicilia F, Brandizzi F, Chung JH, Dupree P. Arabidopsis thaliana expresses multiple Golgi-localised nucleotide-sugar transporters related to GONST1. Mol Gen Genomics. 2004;272:397-410.

31. Bischoff V, Nita S, Neumetzler L, Schindelasch D, Urbain A, Eshed R, Persson S, Delmer D, Scheible WR. TRICHOME BIREFRINGENCE and its homolog AT5G01360 encode plant-specific DUF231 proteins required for cellulose biosynthesis in Arabidopsis. Plant Physiol. 2010;153:590-602.

32. Bischoff $V$, Selbig J, Scheible W-R. Involvement of TBL/DUF231 proteins into cell wall biology. Plant Signal Behav. 2014;5:1057-9.

33. Lusser A, Eberharter A, Loidl A, Goralik-Schramel M, Horngacher M, Haas H, Loidl P. Analysis of the histone acetyltransferase B complex of maize embryos. Nucleic Acids Res. 1999;27:4427-35.

34. Wang Z, Cao H, Chen F, Liu Y. The roles of histone acetylation in seed performance and plant development. Plant Physiol Biochem. 2014;84:12533.

35. Dalisay DS, Kim KW, Lee C, Yang H, Rubel O, Bowen BP, Davin LB, Lewis NG. Dirigent protein-mediated Lignan and Cyanogenic Glucoside formation in flax seed: integrated Omics and MALDI mass spectrometry imaging. J Nat Prod. 2015;78:1231-42

36. De Fhd M-FJ. The seed coat as a modulator of seed-environment relationships in Fabaceae. Braz J Bot. 2001;24:365-75.

37. Radchuk V, Borisjuk L. Physical, metabolic and developmental functions of the seed coat. Front Plant Sci. 2014;5:510.

38. Lopez-Hernandez M, Rodriguez-Alegria ME, Lopez-Munguia A, Wacher C. Evaluation of xylan as carbon source for Weissella spp., a predominant strain in pozol fermentation. LWT-Food Sci Technol. 2018;89:192-7.
39. Dardick C, Callahan AM. Evolution of the fruit endocarp: molecular mechanisms underlying adaptations in seed protection and dispersal strategies. Front Plant Sci. 2014;5:284.

40. Wan L, Li B, Pandey MK, Wu Y, Lei Y, Yan L, Dai X, Jiang H, Zhang J, Wei G, et al. Transcriptome analysis of a new Peanut seed coat mutant for the physiological regulatory mechanism involved in seed coat cracking and pigmentation. Front Plant Sci. 2016;7:1491.

41. Ha B-K, Kim H-K, Kang S-T. Mapping QTLs with epistatic effects and QTL-byenvironment interactions for seed coat cracking in soybeans. Euphytica. 2012;186:933-42.

42. Li N, Li Y. Signaling pathways of seed size control in plants. Curr Opin Plant Biol. 2016;33:23-32.

43. Zhang L, Tan Q, Lee R, Trethewy A, Lee YH, Tegeder M. Altered xylemphloem transfer of amino acids affects metabolism and leads to increased seed yield and oil content in Arabidopsis. Plant Cell. 2010;22:3603-20.

44. Brown RL, Chen ZY, Cleveland TE, Russin JS. Advances in the development of host resistance in corn to Aflatoxin contamination by Aspergillus flavus. Phytopathology. 1999;89:113-7.

45. El-Gebali S, Mistry J, Bateman A, Eddy SR, Luciani A, Potter SC, Qureshi M, Richardson LJ, Salazar GA, Smart A, et al. The Pfam protein families database in 2019. Nucleic Acids Res. 2019;47:D427-32.

46. Mitchell AL, Attwood TK, Babbitt PC, Blum M, Bork P, Bridge A, Brown SD, Chang HY, El-Gebali S, Fraser Ml, et al. InterPro in 2019: improving coverage, classification and access to protein sequence annotations. Nucleic Acids Res. 2019;47:D351-60.

47. Bolger AM, Lohse M, Usadel B. Trimmomatic: a flexible trimmer for Illumina sequence data. Bioinformatics. 2014;30:2114-20.

48. Pertea M, Kim D, Pertea GM, Leek JT, Salzberg SL. Transcript-level expression analysis of RNA-seq experiments with HISAT. StringTie and Ballgown Nat Protoc. 2016;11:1650-67.

\section{Publisher's Note}

Springer Nature remains neutral with regard to jurisdictional claims in published maps and institutional affiliations.

\section{Ready to submit your research? Choose BMC and benefit from:}

- fast, convenient online submission

- thorough peer review by experienced researchers in your field

- rapid publication on acceptance

- support for research data, including large and complex data types

- gold Open Access which fosters wider collaboration and increased citations

- maximum visibility for your research: over $100 \mathrm{M}$ website views per year

At BMC, research is always in progress.

Learn more biomedcentral.com/submissions 